\title{
Cell surface Thomsen-Friedenreich proteome profiling of metastatic prostate cancer cells reveals potential link with cancer stem cell-like phenotype
}

\author{
Feng $\mathrm{Li}^{1,2, *}$, Olga V. Glinskii ${ }^{1,3, *}$, Brian P. Mooney ${ }^{4}$ Kate Rittenhouse-Olson ${ }^{5,6}$, \\ Kenneth J. Pienta7 and Vladislav V. Glinsky ${ }^{1,2}$ \\ ${ }^{1}$ Research Service, Harry S. Truman Memorial Veterans Hospital, Columbia, MO, USA \\ ${ }^{2}$ Department of Pathology and Anatomical Sciences, University of Missouri, School of Medicine, Columbia, MO, USA \\ ${ }^{3}$ Department of Medical Pharmacology and Physiology, University of Missouri, School of Medicine, Columbia, MO, USA \\ ${ }^{4}$ Department of Biochemistry and Charles W. Gehrke Proteomics Center, University of Missouri-Columbia, Columbia, MO, USA \\ ${ }^{5}$ Department of Biotechnical and Clinical Laboratory Sciences and the Department of Microbiology, University at Buffalo, \\ Buffalo, NY, USA \\ ${ }^{6}$ For-Robin, Inc, Buffalo, NY, USA \\ ${ }^{7}$ Department of Urology, The James Buchanan Brady Urological Institute, Departments of Oncology and Pharmacology and \\ Molecular Sciences, The Johns Hopkins School of Medicine, Baltimore, MD, USA \\ *These authors contributed equally to this work
}

Correspondence to: Vladislav V. Glinsky, email: glinskiivl@health.missouri.edu

Keywords: Thomsen-Friedenreich antigen, proteomics, tumor metastasis, cancer stem cells, prostate cancer

Received: June 02, $2017 \quad$ Accepted: September 29, $2017 \quad$ Published: October 20, 2017

Copyright: Li et al. This is an open-access article distributed under the terms of the Creative Commons Attribution License 3.0 (CC BY 3.0 ), which permits unrestricted use, distribution, and reproduction in any medium, provided the original author and source are credited.

\section{ABSTRACT}

The tumor-associated Thomsen-Friedenreich glycoantigen (TF-Ag) plays an important role in hematogenous metastasis of multiple cancers. The LTQ Orbitrap LC-MS/MS mass spectrometry analysis of cell surface TF-Ag proteome of metastatic prostate cancer cells reveals that several cell surface glycoproteins expressing this carbohydrate antigen in prostate cancer (CD44, a2 integrin, $\beta 1$ integrin, CD49f, CD133, CD59, EphA2, CD138, transferrin receptor, profilin) are either known as stem cell markers or control important cancer stem-like cell functions. This outcome points to a potential link between TF-Ag expression and prostate cancer stem-like phenotype. Indeed, selecting prostate cancer cells for TF-Ag expression resulted in the enrichment of cells with stem-like properties such as enhanced clonogenic survival and growth, prostasphere formation under non-differentiating and differentiating conditions, and elevated expression of stem cell markers such as CD44 and CD133. Further, the analysis of the recent literature demonstrates that TF-Ag is a common denominator for multiple prostate cancer stem-like cell populations identified to date and otherwise characterized by distinct molecular signatures. The current paradigm suggests that dissemination of tumor cells with stem-like properties to bone marrow that occurred before surgery and/or radiation therapy is largely responsible for disease recurrence years after radical treatment causing a massive clinical problem in prostate cancer. Thus, developing means for destroying disseminated prostate cancer stem-like cells is an important goal of modern cancer research. The results presented in this study suggest that multiple subpopulation of putative prostate cancer stem-like cells characterized by distinct molecular signatures can be attacked using a single target commonly expressed on these cells, the TF-Ag. 


\section{INTRODUCTION}

Thomsen-Friedenreich antigen (TF antigen, TF$\mathrm{Ag}, \mathrm{T}$ antigen, $\mathrm{CD} 176)$ is a core 1 glycan structure Gal $\beta 1-3$ GalNAc $\alpha 1$ - O-linked to serine or threonine, which is commonly present in a cryptic form on many normal cell types, but is unmasked and reactive on the vast majority (about $90 \%$ ) of all human cancers including colon, breast, bladder, prostate, liver, ovary and stomach [1]. Previous works from this and other groups demonstrated the role for TF-Ag in metastatic spread of breast $[2,3]$, prostate $[2,4,5]$, colon [6], and pancreatic cancer [7]. Specifically, TF-Ag has been implicated in mediating metastatic cell adhesive interactions with vascular endothelium $[2,6,8,9]$ as well as homotypic tumor cell aggregation [10], thus controlling important rate-limiting steps in cancer metastasis [11]. Two recent groundbreaking studies highlight the critical role played in estrogen receptor-negative breast cancer metastasis by the enzyme ST6GalNAc2 controlling the expression of TF-antigen [12], and by C2GnT2 and ST6GalNAc4 glycosyltransferase activity in lung adenocarcinoma controlling metastatic cell attachment to galectin-3presenting cells in the metastatic niche via regulation of $\mathrm{TF}$ antigen expression [13].

To date, in various types of cancer $\mathrm{TF}$ antigen has been shown to be expressed on several glycoproteins. Specifically, the mucin MUC1 has been shown to express TF-Ag in breast [14] and colon [6] while MUC4 in pancreatic [7] cancer; CD44 has been identified as a major TF-Ag carrier in colon cancer [15]; in cancers of lung, breast, and liver CD133 and CD44 express TF-Ag [16]; and CD34 was shown to express TF-Ag on malignant hematopoietic cells [17]. In prostate cancer, however, the TF proteome has not been investigated systematically until now. Thus, in this study we have performed the analysis of cell surface prostate cancer TF proteome. Using the affinity purification of biotinylated cell surface proteins followed by a pull-down with TF-Ag specific peanut agglutinin (PNA) and a shotgun mass spectrometry analysis as well as immunoprecipitation (IP) of individual glycoproteins, and Western blot verification of TF-Ag expression, we unambiguously identified 18 cell surface glycoproteins as TF-Ag carriers expressed on PC-3 and DU-145 metastatic human prostate carcinoma cells. Remarkably, several of these TF-Ag expressing glycoproteins namely CD44, $\alpha 2 \beta 1$ integrin, CD133, CD49f ( $\alpha 6$ integrin), and ephrin type-A receptor 2 (EphA2) either serve as most commonly used cell surface prostate cancer stem cell markers (CD44, a2 $\beta 1$ integrin, CD133, CD49f) or control stem cell like functions including clonogenic potential, self-renewal, prostasphere formation, tumor onset, and dissemination of prostate carcinoma to the skeleton (EphA2) [18-27].

Indeed, selecting $\mathrm{TF}-\mathrm{Ag}$ expressing $\mathrm{PC}-3$ prostate cancer cells yielded a subpopulation of cells with enhanced stem cell properties such as clonogenic survival, clonogenic growth, prostasphere formation under both non-differentiating and differentiating conditions, and enhanced expression of stem cell markers compared with their TF-Ag negative counterparts. Taken together, these results strongly suggest that TF antigen expression in prostate cancer cells is associated with the stem cell like phenotype. It appears that TF antigen could be a common denominator for multiple populations of prostate cancer stem like cells identified to date and otherwise characterized by distinct cell surface signatures [18]. Consequently, TF antigen specific therapeutics such as anti-TF antibodies [28] or TF-Ag binding peptides [29] could potentially provide means for targeting simultaneously multiple prostate cancer stem cell subtypes.

\section{RESULTS}

\section{Cell surface metastatic prostate cancer cell TF proteome}

\section{Two-step affinity purification and LTQ orbitrap LC-MS/MS analysis of cell surface TF-ag expressing glycoproteins}

In the present work, we employed a method combining biotinylation of cell surface proteins followed by a streptavidin agarose pull-down, lectin affinity chromatography, and LC-MS/MS mass spectrometry analysis to identify cell surface TF-Ag glycoprotein carriers expressed on PC-3 and DU-145 human prostate carcinoma cell lines.

The cell surface proteins were first labeled by SulfoNHS-SS-Biotin, followed by the affinity purification using streptavidin-coated agarose beads. Next, upon reduction with DTT, disulfide bonds were broken to release biotinylated proteins from the beads [30], which were further purified using affinity chromatography with peanut agglutinin (PNA-lectin), having high specific capability to bind peptides with TF-Ag glycan structure, to capture the TF-Ag expressing glycoproteins.

The enriched TF-Ag carrying glycoproteins were digested and analyzed by LC-MS/MS using LTQ Orbitrap mass spectrometry. Figure 1 shows representative base peak chromatograms of the PNA lectin captured fractions from PC-3 and DU-145 cell surface proteins, as well as examples of MS/MS spectra of the same peptides from CD44, $\alpha 2$ integrin, $\beta 1$ integrin, and $\alpha 6$ integrin identified in samples from PC-3 and DU-145 cells.

In three independent experiments, the LTQ Orbitrap analysis resulted in the identification of 45 , 152 , and 97 proteins from biotinylated PNA-lectin tandem affinity purification fractions from DU145 cells. Similarly, 123, 39, 102, and 102 proteins were identified in four independent experiments with PC-3 cells. However, due to the limitations of 
immunoprecipitation technique and high sensitivity of the LTQ Orbitrap mass spectrometry, not all of the proteins identified in these experiments are cell surface TF-Ag carriers. A significant number of them could be either non-specifically absorbed high abundance intracellular proteins or co-precipitated proteins interacting with the TF-Ag expressing glycopeptides. Thus, our next task was to screen out such proteins and select only cell surface glycoproteins. To achieve this, a consensus transmembrane prediction strategy combining the prediction results from several different transmembrane prediction software tools was used.


\section{Consensus transmembrane prediction analysis}

A consensus prediction strategy employing several transmembrane prediction tools has been proven to improve overall accuracy compared to any of the individual methods. [31]. Here, three transmembrane prediction software tools were used, including ones based upon simple hydrophobicity analysis (TopPred), thermodynamic and biological principles (MPEx), and hidden Markov models (TMHMM) [32-34]. First, one dataset generated from DU-145 cell line experiment number two including 152 identified proteins was used
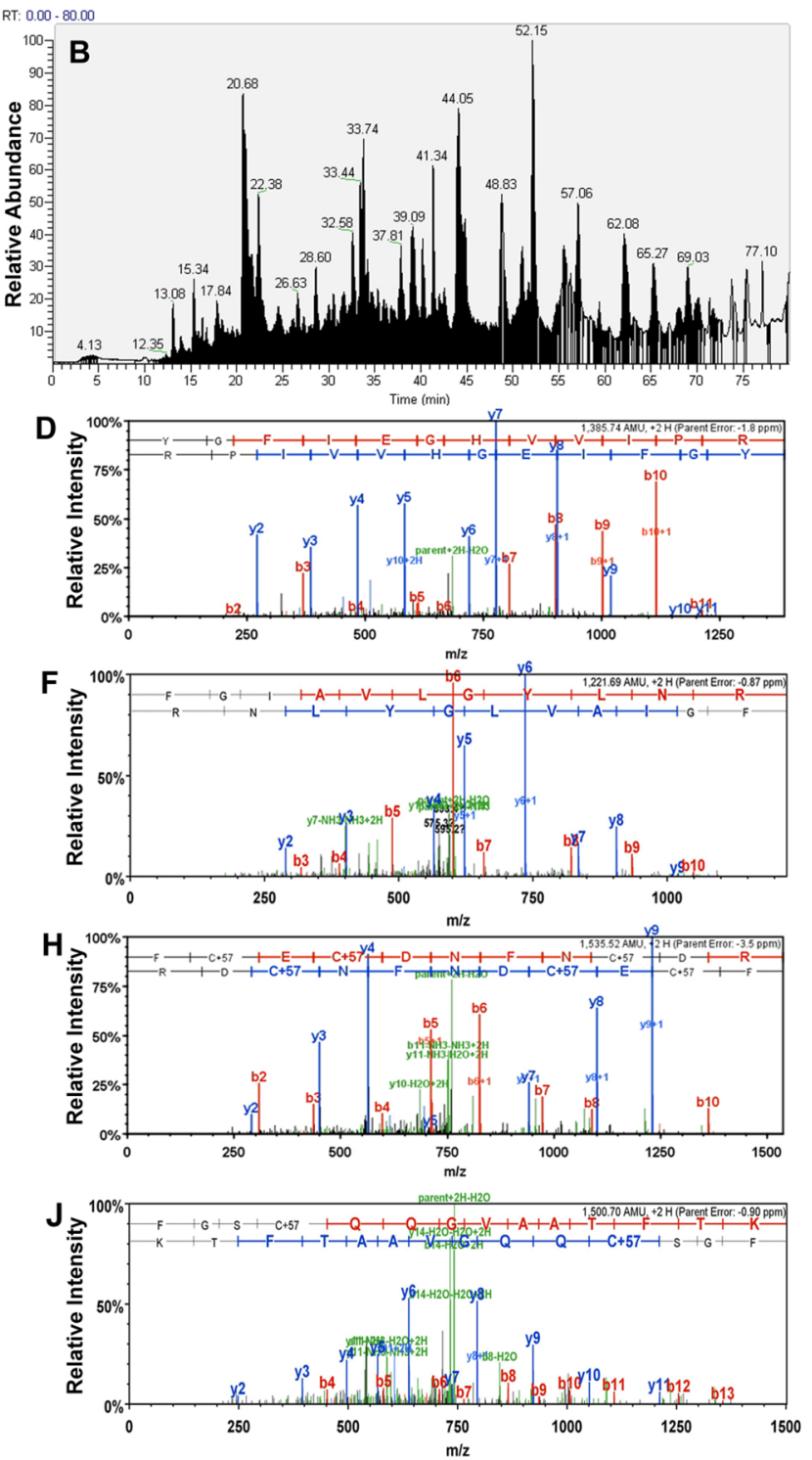

Figure 1: Shotgun proteomics and LC MS/MS analysis. (A) and (B), Representative base peak chromatograms of PNA-lectin captured fraction from DU-145 (A) and PC-3 (B) cells. (C) and (D), Examples of MS/MS spectra of the same peptide from CD44 identified in DU-145 (C) and PC-3 (D) cells. The sequence of the peptide was identified as YGFIEGHVVIPR. (E) and (F), MS/MS spectra of the same peptide from the integrin alpha-2 identified in DU-145 (E) and PC-3 (F) cells. The sequence of the peptide was identified as FGIAVLGYLNR. (G) and (H), MS/MS spectra of the same peptide from the integrin beta-1 identified in DU-145 (G) and PC-3 (H) cells. The sequence of the peptide was identified as FCECDNFNCDR. (I) and (J), MS/MS spectra of the same peptide from the integrin alpha-6 identified in PC-3 (I) and DU-145 (J) cells. The sequence of the peptide was identified as FGSCQQGVAATFTK. 
to evaluate the accuracy of these three transmembrane prediction software tools. All software tools were set up to the default settings. Compared to MPEx and TopPred, TMHMM predicted 18 transmembrane proteins with at least one transmembrane segment, which was the least number of predicted transmembrane proteins among the three prediction results. MPEx and TopPred had significantly more predicted transmembrane proteins with at least one transmembrane segment. However, the analysis yielded almost the same number of transmembrane proteins when the threshold was set to two transmembrane segments in MPEx and three transmembrane segments in TopPred. TMHMM had the highest conservation and accuracy, which was coincident with the literature. Thus, for the final analysis, the threshold of transmembrane proteins was set to one transmembrane segment in TMHMM, two transmembrane segments in MPEx and three transmembrane segments in TopPred in all datasets.

Although TMHMM has the highest accuracy to predict transmembrane proteins among these three prediction software tools, the consensus strategy can provide a better result than any single prediction method alone. For example, isoform 1 of plectin was predicted as a non-transmembrane segment containing by TMHMM, but as having two transmembrane segments by MPEx and three transmembrane segments by TopPred. Thus, based on the consensus prediction strategy, plectin should be considered as protein with transmembrane segments, which is consistent with the published literature [35]. All positive transmembrane prediction results were manually validated and imported into a desktop Microsoft Access database. The proteins that appeared at least twice in all datasets were considered TF-antigen expressing cell surface proteins and listed in Figure 2A. The proteins appearing in all datasets only once were considered as possible TF-antigen expressing proteins and listed in Supplemental Table 1 as well as TF-Ag interacting and mucin-like proteins identified by the LTQ Orbitrap analysis.

One remarkable outcome of this analysis was that a significant fraction of glycoproteins identified as cell surface TF-antigen carriers in PC-3 and DU-145 cells (Figure 2A) are associated with stem cell phenotype or function, including prostate cancer stem-like cells. Specifically, CD44, $\alpha 2$ integrin, $\beta 1$ integrin, and CD49f ( $\alpha 6$ integrin) are the most commonly recognized prostate cancer stem cell markers, while EphA2 controls prostate cancer stem cell-like functions such as clonogenic potential, self-renewal, prostasphere formation, tumor onset, and dissemination of prostate carcinoma to the bone [18-27], and syndecan-1 (CD138) stabilizes prostate cancer tumor-initiating cells [36]. In addition, CD59 is one of the most commonly reported positive markers for adipose-derived stem cells [37] and human mesenchymal stem cells [38]; transferrin receptor is preferentially required and overexpressed by glioblastoma stem-like cells [39]; and profilin is required for germline stem cell maintenance [40] and promotes migration, invasion, and stemness of human colorectal cancer stem cells [41].

\section{Western blot verification of TF-Ag expression}

To validate the mass spectrometry identified TF-Ag expressing cell surface proteins, eight of the identified glycoproteins (integrin $\alpha 2$, integrin $\beta 1$, integrin $\alpha 6$, CD98, CD44, Syndecan-1, TFRC and CD59) were individually immunoprecipitated, resolved by SDS PAGE, and probed with anti-TF-Ag monoclonal antibody JAA-F11 [42] using Western blot techniques. In addition, yet another commonly recognized stem cell marker (including prostate cancer stem cells) CD133, which is expressed at rather low levels and could be often masked by more abundant proteins in shotgun mass spectrometry analyses, was also immunoprecipitated and analyzed for TF antigen expression by Western blot. After probing with TF-Ag-specific antibody JAA-F11, the membranes were stripped and reprobed with corresponding protein-specific antibodies to confirm the correct position of TF-Agpositive bands. The results of these experiments confirmed that all selected glycoproteins including CD133 do indeed express TF antigen (Figure 2B).

\section{Selection and characterization of TF antigen positive prostate cancer cells}

TF antigen positive prostate cancer cells exhibit enhanced clonogenic survival and clonogenic growth ability compared to their TF antigen negative counterparts

The fact that the majority of prostate cancer cell surface TF-Ag-expressing glycoproteins are known as stem cell markers prompted us to ask a question whether selecting TF-Ag-positive prostate cancer cells will enrich for the cells with stem-like properties. To interrogate this question, we have used magnetic activated cell sorting (MACS) with biotinylated anti-TF-Ag antibody JAA-F11 to isolate TFAg-positive (TF+) and TF-Ag-negative (TF-) prostate cancer cells. Of note, in multiple isolation experiments, the average yield of TF+ cells was 3.93\% $\pm 0.901 \%$ for PC-3 and $0.36 \%$ $\pm 0.21 \%$ for DU-145 cells (mean \pm standard error of mean).

One of the fundamental unique properties attributed to stem cells, including prostate cancer stem-like cells, is self-renewal. There are several commonly accepted tools for analyzing putative stem-like cells self-renewal capacity including clonogenic assay, which is based on seeding a small number of cells and monitoring colony formation over a defined time period. Remarkably, in contrast to TF- cells, the $\mathrm{TF}+$ population exhibited clonogenic growth patterns even when plated at the regular densities and culturing conditions (Figure 3A and 3B). When plated at the low density (200 cells per well in a 24-well plate) for clonogenic survival and growth, TF+ PC-3 cells formed 3.5-fold more clones than TFcells did, (Figure 3C). Similarly, TF+ DU-145 cells exhibited 
stronger propensity to clonogenic survival and growth than their TF- counterparts (Figure 3D and 3E) indicating that TF-Ag selection does enrich for prostate cancer cells with enhanced clonogenicity.

TF antigen positive prostate cancer cells form significantly more and larger prostaspheres under both non-differentiating and differentiating conditions

Another approach to analyzing self-renewal capacity of cancer stem-like cells is the sphere formation assay. The sphere formation assay is based on low adherence cultures in defined serum-free medium (non-differentiating conditions), or serum-supplemented medium (differentiating conditions) to produce multicellular spheroid aggregates called tumorspheres (prostaspheres in prostate cancer), which are enriched for cells with stem-like phenotypes.

In our experiments, under non-differentiating conditions (serum free cultures in Matrigel) $\mathrm{TF}+\mathrm{PC}-3$ cells formed greater than 2-fold more prostaspheres of a much bigger size than TF- cells did (Figure 3G-3I). Under differentiating conditions (complete media in ultra-low attachment plates), TF+ PC-3 cells also formed greater than 2-fold more prostaspheres than TF- cells of even bigger size (Figure 3J-3L). Similarly, TF+ DU-145 cells formed 2-fold

A

Cell Surface TF-Antigen Expressing Glycoproteins

\begin{tabular}{|c|c|c|}
\hline $\begin{array}{c}\text { Accession } \\
\text { Number }\end{array}$ & Protein Name & Gene \\
\hline IPI00002230.6 & Neutral Cholesterol Ester Hydrolase 1 Isoform B & NCEH1 \\
\hline IPI00002441.2 & Syndecan-1 (CD138) & SDC1 \\
\hline IPI00006482.1 & Isoform Long of Sodium/Potassium Transporting ATPase Subunit Alpha 1 & ATP1A1 \\
\hline IPI00010697.2 & Is of orm Alpha-6X1X2B of Integrin Alpha-6 & ITGA6 \\
\hline IPI0 0011302.1 & CD59 Glycoprotein & CD59 \\
\hline IPI00013744.1 & Integrin Alpha-2 & ITGA2 \\
\hline IPI00014898.3 & Isoform 1 of Plectin & PLEC \\
\hline IPI00018452.3 & Copine -1 & CPNE1 \\
\hline IPI00021048.1 & Isoform 1 of Myoferlin & MYOF \\
\hline IPI00021267.2 & Ephrin Type A Receptor 2 & EPHA2 \\
\hline IPI00022462.2 & Transferrin Receptor Protein 1 & TFRC \\
\hline IPI0 0027493.1 & Isoform 2 of 4F2 Cell-Surface Antigen Heavy Chain (CD98) & SLC3A2 \\
\hline IPI00107555.5 & Profilin & PFN2 \\
\hline IPI0 0217561.2 & Is of orm Beta-1C of Integrin Beta-1 & ITGB1 \\
\hline IPI00217563.4 & Is of orm Beta-1A of Integrin Beta-1 & ITGB1 \\
\hline IPI00289334.2 & Isoform 1 of Filamin-B & FLNB \\
\hline IPI00297160.4 & Is of orm 12 of CD44 Antigen & CD44 \\
\hline IPI00472013.1 & HLA Class I Histocompatibility Antigen A-33 Alpha Chain & HLA-A33 \\
\hline
\end{tabular}

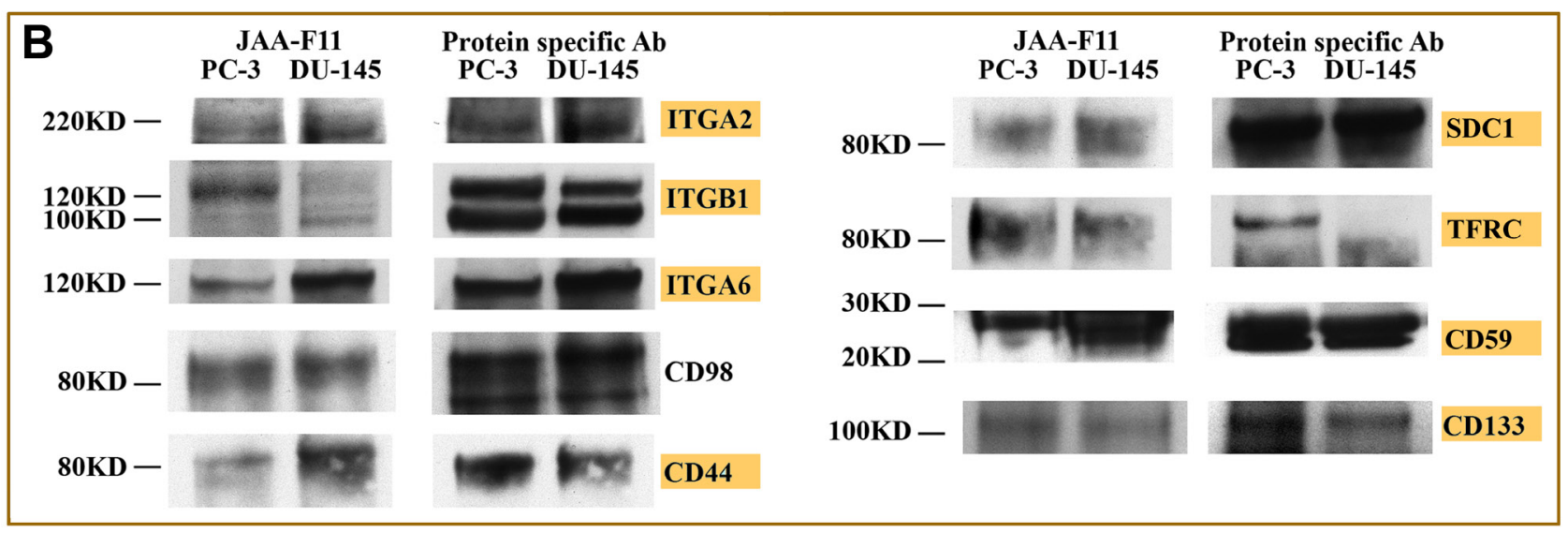

Figure 2: Identification of cell surface glycoproteins expressing TF-Ag in prostate cancer. (A) List of cell surface glycoproteins expressing TF-Ag identified by LTQ Orbitrap LC MS/MS analysis of PNA-labeled fractions from DU-145 and PC-3 cells. Glycoproteins highlighted in bold are known as prostate cancer stem cell markers or controlling important cancer stem-like cell functions. (B) Confirmation of TF-Ag expression by IP and Western analysis. Individual glycoproteins from PC-3 and DU-145 cells were immunoprecipitated, resolved by PAG electrophoresis, transferred to the nitrocellulose membrane, and probed with anti-TF-Ag mAb JAA-F11 for TF-Ag expression. To verify a proper position of TF-Ag-reactive bands, membranes were stripped and reprobed with protein-specific Abs used for IP. Names of the proteins known as prostate cancer stem cell markers or controlling important cancer stem-like cell functions are highlighted in yellow. 
more prostaspheres than TF- cells (Figure 3F). The results of these experiments demonstrated that $\mathrm{TF}+$ cells possess far superior prostasphere forming ability compared to TF- cells.

\section{TF antigen positive prostate cancer cells express higher levels of stem cell markers}

As TF+ prostate cancer cells demonstrated much higher ability for clonogenic survival and growth, as well as the propensity to form prostaspheres under both differentiating and non-differentiating conditions compared to TF- cells, our next question was whether TFAg selection leads to the enrichment of cells with elevated expression of stem cell markers such as CD44 and CD133. The comparison of CD44 and CD133 expression in TF+ and TF- cells by Western blot analysis revealed that TF+ cells express significantly higher levels of both CD44 and CD133 (Figure 3M). Further, when we reversed the experiment and selected using MACS techniques CD44 positive prostate cancer cells, the immunofluorescent analysis demonstrated that practically $100 \%$ of CD44+ cells express TF-Ag (Figure $3 \mathrm{~N}$ and $3 \mathrm{O}$ ).

\section{DISCUSSION}

The results of LTQ Orbitrap LC-MS/MS mass spectrometry analysis of prostate cancer cell surface TFAg proteome presented in this study demonstrate that many of TF-Ag expressing cell surface glycoproteins in prostate cancer cells are in fact either well known stem cell markers (CD44, $\alpha 2$ integrin, $\beta 1$ integrin, CD49f, CD59) or the proteins controlling important cancer stemlike cell functions (EphA2, CD138, transferrin receptor, profilin). This outcome strongly suggests that there is a link between TF-Ag expression and prostate cancer stemlike phenotype. Indeed, selecting prostate cancer cells for TF-Ag expression resulted in the enrichment of cells with stem-like properties characterizing self-renewal capacity such as enhanced clonogenic survival and growth (Figure $3 \mathrm{~A}-3 \mathrm{E}$ ) and prostasphere formation under both nondifferentiating and differentiating conditions (Figures $3 \mathrm{~F}-3 \mathrm{~L})$, as well as increased expression of stem cell markers CD44 and CD133 (Figure 3M). Obviously, there are multiple mechanistic questions to be answered regarding the association between TF-Ag expression and cancer stem-like phenotype. However, there is one practical implication of this study requiring an immediate attention. The fact that various stem cell markers in prostate cancer express TF-Ag offers unparalleled opportunity for therapeutic targeting of multiple subpopulations of prostate cancer stem-like cells. Indeed, a simple analysis of the cell surface signatures discovered in recent years for putative prostate cancer stem cells by different groups (Table 1) reveals that every one of these signatures contains at least one, or two, or even three TF-Ag glycoprotein carriers.
Finding efficient approaches to targeting prostate cancer stem-like cells is an important challenge of modern cancer research. In the recent review, Yu et al. indicate that "Each year, 40,000 men who 'should' have been cured of their PCa [prostate cancer] by surgery or radiation therapy present with incurable metastatic disease that will manifest itself as metastatic lesions in the bone, usually years after primary treatment" [43]. The best currently available explanation for this is that tumor cell dissemination to the bone marrow microenvironment occurred before primary tumor removal, and disseminated tumor cells with cancer stem cell properties are responsible for a disease recurrence [43]. Consequently, eliminating prostate cancer stem-like cells may offer the potential of completely eradicating the disease [44]. Hence, defining specific cell surface markers or pathways associated with prostate cancer stem-like cells, which could potentially serve as therapeutic targets, is critical for achieving this goal [44]. However, a simple look at the currently known cell surface signatures for putative prostate cancer stem-like cells (Table 1) demonstrates that using the markers involved straightforward as potential therapeutic targets could be somewhat problematic as the same molecules are often expressed on multiple normal cell types including normal stem cells as well. Here, the fact that in prostate cancer cell surface stem cell markers such as CD44, $\alpha 2 \beta 1$ integrin, CD133, CD49f ( $\alpha 6$ integrin) are all decorated with TF-Ag, which is covalently masked (either sialylated or further glycosylated) on normal cells, provides a unique opportunity to discriminate between these cell surface markers expressed on cancer stem-like cells and their normal counterparts and target prostate cancer stem cells via the TF-Ag. Further, as TF-Ag appears to be a "common denominator" for multiple prostate cancer stem-like cell populations otherwise characterized by distinct molecular signatures (Table 1), targeting prostate cancer stem-like cells via TF-Ag will endow an opportunity of attacking various species of cancer stem cells at once.

\section{MATERIALS AND METHODS}

\section{Cell lines and cultures}

Human prostate carcinoma cell lines PC-3 and DU145 were from ATCC (Manassas, VA, USA). The identity of cell lines was confirmed by IDEXX BioResearch using short tandem repeat (STR) profile following the completion of the experiments. Both cell lines were routinely maintained on plastic in $5 \% \mathrm{CO}_{2}$ humidified atmosphere using RPMI-1640 medium supplemented with L-glutamine, 10\% FBS, sodium pyruvate, and nonessential amino acids. 

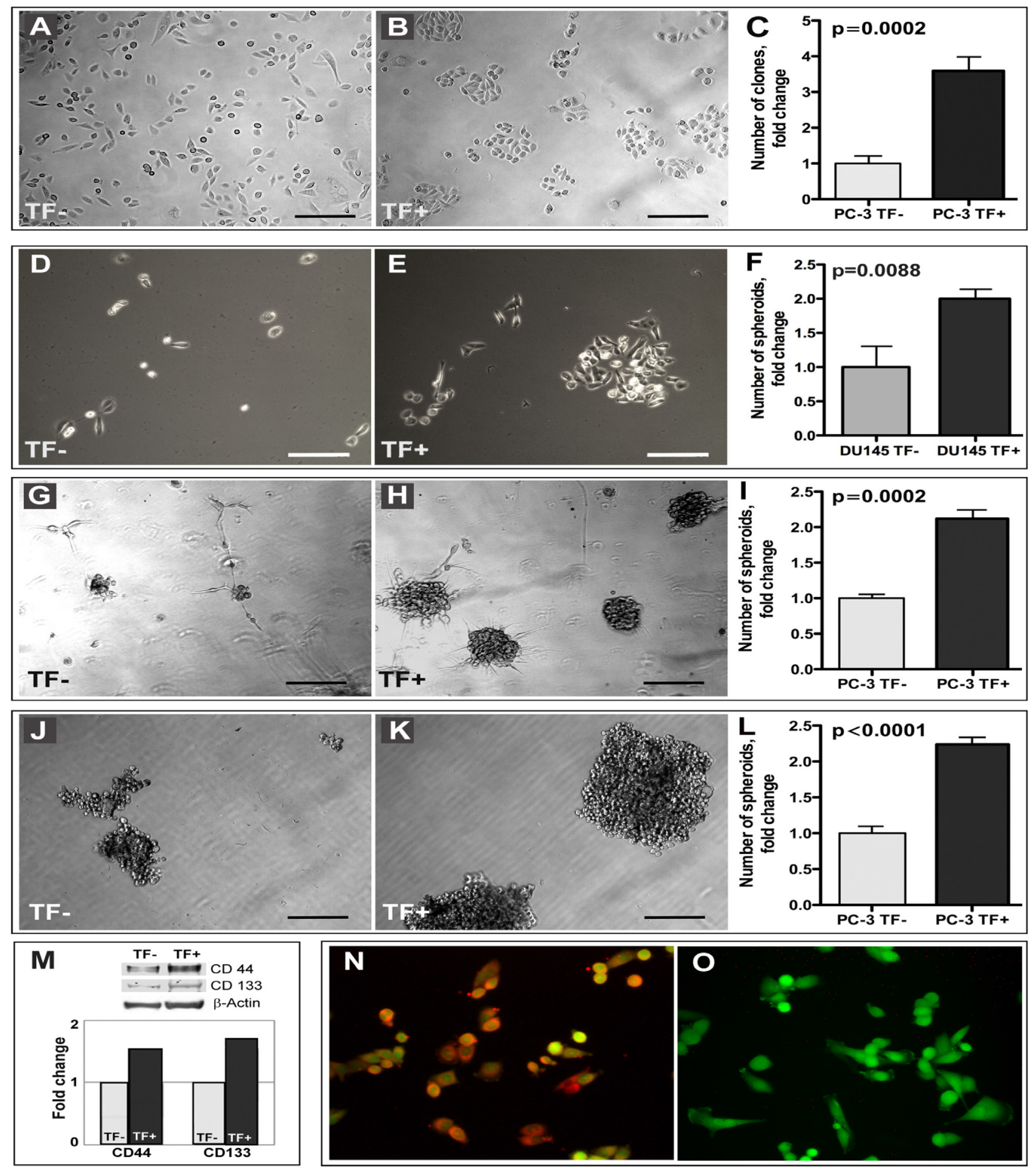

Figure 3: Stem-like properties of TF+ cells. Unlike TF- cells (A), TF+ population $(\mathbf{B})$ of PC-3 cells exhibits growth patterns characteristic of clonogenic growth even when plated at the regular densities and culturing conditions. (C) TF+ PC-3 cells form 3.5-fold more clones than TF- cells, when plated at the low density (200 cells per well in a 24-well plate) for clonogenic survival. Similarly, TF+ DU-145 cells exhibit enhanced ability to clonogenic survival and growth compared to TF- cells (D) and (E). TF+ DU-145 cells form 2-fold more prostaspheres than their TF- counterparts (F). Compared to TF- cells (G), TF+ population (H) of PC-3 cells forms $>2$-fold more prostaspheres (I) of a much bigger size, when cultured for 2 weeks in Matrigel using serum free media. Compared to TF- cells (J), $\mathrm{TF}+$ population $(\mathbf{K})$ of PC-3 cells forms $>2$-fold more prostaspheres $(\mathbf{L})$ of a much bigger size, when cultured for 2 weeks in ultra-low attachment plates using complete media. (M) Western blot analysis of TF+ and TF-.PC-3 cells. Note higher expression of stem cell markers CD44 and CD133 by TF+ cells. (N and $\mathbf{O}$ ) Immunofluorescent analysis of TF-Ag expression on CD44 positive subpopulation of PC-3 cells using anti-TF-Ag antibody JAA-F11. N, 100\% of CD44+ cells express TF-Ag. O, Negative control omitting primary antibody. In K and L, red - TF-Ag; green - GFP. In C, F, I, L, and M, the results are normalized to TF- cells. In A, B, D, E, G, H, J and K, bar, $200 \mu$ m. 
Table 1: Cell surface marker signatures used recently to characterize putative prostate cancer CSC populations (markers in bold express TF-Ag in prostate cancer cells)

\begin{tabular}{|c|c|c|}
\hline Signature & CSC Source/Model & Reference \\
\hline $\mathrm{CD} 44+/ \mathrm{CD} 24^{-}$ & Cell lines and xenografts & Hurt EM et al. British Journal of Cancer 2008, 98:756-765 [46] \\
\hline $\mathrm{CD} 44^{+} / \alpha 2 \beta 1^{\mathrm{hi}} / \mathrm{CD} 133^{+}$ & Primary tumors & Collins AT et al. Cancer Res 2005; 65:10946-10951 [27] \\
\hline $\mathrm{CD} 44^{+} / \alpha 2 \beta 1^{+}$ & DU145, LAPC4, LAPC9 & Patrawala L et al. Cancer Res 2007; 67:6796-6805 [47] \\
\hline $\mathrm{CD} 133^{\mathrm{hi} /} / \mathrm{CD} 44^{\mathrm{hi}} / \mathrm{OCT} 4^{\mathrm{hi}}$ & NHPrE1 & Jiang M et al. Stem Cells 2010; 28:344-56 [48] \\
\hline CD49f fhi $^{\text {Trop }} 2^{\text {hi }}$ & Primary prostate tissue & Goldstein AS et al. Science 2010; 329:568-571 [49] \\
\hline $\mathrm{Lin}^{-} / \mathrm{Sca}-1^{-} / \mathrm{CD} 49 \mathrm{f}^{\mathrm{hi}}$ & $\mathrm{cPten}^{-/}$ & $\begin{array}{l}\text { Lawson DA et al. Proc Natl Acad Sci USA 2010; 107:2610-5 [50]; } \\
\text { Liao CP et al. Cancer Res 2010; 70:7294-7303 [51] }\end{array}$ \\
\hline $\begin{array}{l}\mathrm{CD} 44^{+} / \mathrm{CD} 133^{+} / \mathrm{ABCG}^{+} / \\
\mathrm{CD} 24^{-}\end{array}$ & Primary tumors & Patrawala L et al. Cancer Res 2005, 65:6207-6219 [52] \\
\hline $\begin{array}{l}\mathrm{PSA}^{- \text {llow }} / \mathrm{ALDH}^{+} / \mathrm{CD} 44^{+} / \\
\alpha 2 \beta 1^{+}\end{array}$ & Cell lines and xenografts & Salvatori L et al. PLoS One. 2012; 7(2):e31467 [53] \\
\hline
\end{tabular}

Isolation of cell surface TF-Ag expressing glycoproteins

For each experiment, cell surface proteins in four T75 flasks of $70-80 \%$ confluent live cultures of either PC-3 or DU-145 cells were biotinylated and purified using Pierce Cell Surface Protein Isolation Kit (Thermo Fisher Scientific Inc., Rockford, IL) according to the manufacturer instructions. Next, TF-Ag expressing glycoproteins were enriched using TF-Ag specific PNA (peanut agglutinin) lectin affinity chromatography (see Supplementary Methods for detail).

\section{LTQ orbitrap mass spectrometry and data analysis}

For mass spectrometric analysis, isolated cell surface glycoproteins were concentrated, digested with mass spectrometry grade trypsin gold (Promega, Madison, WI) and further purified using C18 zip-tips. Tryptic peptides were loaded onto a C8 trap column (C8 CapTrap, Michrom Bioresources) and analyzed on a system with Proxeon Easy nLC system attached to the LTQ Orbitrap mass spectrometer using CID and ETD fragmentation. All MS/ MS spectra were searched against the IPI database (IPI human_20090713.fasta) using SEQUEST TM algorithm on the Sorcerer 2 integrated data appliances (IDA) server with default peak list extraction parameters. Post-search analysis was performed using the Scaffold, implementing PeptideProphet and ProteinProphet algorithms.

\section{Bioinformatic transmembrane topology prediction and data integration}

The consensus transmembrane topology prediction strategy was adopted and three transmembrane prediction software tools including TMHMM 2.0 (http://www.cbs.dtu. dk/services/TMHMM-2.0/), MPEx 3.2 and TopPred 0.01 (http://mobyle.pasteur.fr/cgi-bin/portal.py?\#forms::toppred) were used to identify the transmembrane proteins (see Supplementary Methods for detail).

\section{Isolation and analysis of TF-Ag positive and TF- Ag negative cells}

Magnetic activated cell sorting (MACS) with biotinylated anti-TF-Ag antibody JAA-F11 [42] was used to isolate TF-Ag positive (TF+) and TF-Ag negative (TF-) prostate cancer cells (see Supplementary Methods for detail). Clonogenic survival and growth of TF+ and TF- cells was assessed as previously described [10]. Prostasphere formation of $\mathrm{TF}+$ and TF- cells under non-differentiating conditions was analyzed exactly as described elsewhere [45] using $3 \times 10^{3}$ cells per well in 12-well plates. For prostasphere formation under differentiating conditions, cells were plated and cultured as described in Supplementary Methods.

\section{Immunofluorescence and western blot analysis}

CD44 positive prostate cancer cells were isolated by MACS using CD44 MicroBeads (Miltenyi Biotech, San Diego, CA; 130-095-194) and probed for TF-Ag expression using anti-TF-Ag antibody JAA-F11 and goat anti-mouse Alexa Fluor 594 conjugated antibody (Molecular Probes by Life Technologies, Thermo Fisher Scientific, Waltham, MA; Cat \# A11020). For Western blot analysis, see complete list of antibodies used in Supplementary Methods.

\section{CONFLICTS OF INTEREST}

The authors declare no potential conflicts of interest.

\section{GRANT SUPPORT}

This research was supported in part by the Award \#1I01BX000609 from the Biomedical Laboratory 
Research \& Development Service of the VA Office of Research and Development (VVG) and the National Cancer Institute of the National Institutes of Health Award \#R01CA160461 (VVG).

\section{REFERENCES}

1. Yu LG. The oncofetal Thomsen-Friedenreich carbohydrate antigen in cancer progression. Glycoconj J. 2007; 24:411-420.

2. Glinsky VV, Glinsky GV, Rittenhouse-Olson K, Huflejt ME, Glinskii OV, Deutscher SL, Quinn TP. The role of ThomsenFriedenreich antigen in adhesion of human breast and prostate cancer cells to the endothelium. Cancer Res. 2001; 61:4851-4857.

3. Heimburg J, Yan J, Morey S, Glinskii OV, Huxley VH, Wild L, Klick R, Roy R, Glinsky VV, Rittenhouse-Olson $\mathrm{K}$. Inhibition of spontaneous breast cancer metastasis by anti-Thomsen-Friedenreich antigen monoclonal antibody JAA-F11. Neoplasia. 2006; 8:939-948.

4. Glinskii OV, Sud S, Mossine VV, Mawhinney TP, Anthony DC, Glinsky GV, Pienta KJ, Glinsky VV. Inhibition of prostate cancer bone metastasis by synthetic TF antigen mimic/galectin-3 inhibitor lactulose-L-leucine. Neoplasia. 2012; 14:65-73.

5. Guha P, Kaptan E, Bandyopadhyaya G, Kaczanowska S, Davila E, Thompson K, Martin SS, Kalvakolanu DV, Vasta GR, Ahmed H. Cod glycopeptide with picomolar affinity to galectin-3 suppresses T-cell apoptosis and prostate cancer metastasis. Proc Natl Acad Sci U S A. 2013; 110:5052-5057.

6. Yu LG, Andrews N, Zhao Q, McKean D, Williams JF, Connor LJ, Gerasimenko OV, Hilkens J, Hirabayashi J, Kasai K, Rhodes JM. Galectin-3 interaction with ThomsenFriedenreich disaccharide on cancer-associated MUC1 causes increased cancer cell endothelial adhesion. J Biol Chem. 2007; 282:773-781.

7. Senapati S, Chaturvedi P, Chaney WG, Chakraborty S, Gnanapragassam VS, Sasson AR, Batra SK. Novel Ineraction of MUC4 and galectin: potential pathobiological implications for metastasis in lethal pancreatic cancer. Clin Cancer Res. 2011; 17:267-274.

8. Glinskii OV, Huxley VH, Glinsky GV, Pienta KJ, Raz A, Glinsky VV. Mechanical entrapment is insufficient and intercellular adhesion is essential for metastatic cell arrest in distant organs. Neoplasia. 2005; 7:522-527.

9. Gassmann P, Kang ML, Mees ST, Haier J. In vivo tumor cell adhesion in the pulmonary microvasculature is exclusively mediated by tumor cell--endothelial cell interaction. BMC Cancer. 2010; 10:177. https://doi.org/10.1186/1471-240710-177.

10. Glinsky VV, Glinsky GV, Glinskii OV, Huxley VH, Turk JR, Mossine VV, Deutscher SL, Pienta KJ, Quinn TP. Intravascular metastatic cancer cell homotypic aggregation at the sites of primary attachment to the endothelium. Cancer Res. 2003; 63:3805-3811.
11. Glinsky VV. Intravascular cell-to-cell adhesive interactions and bone metastasis. Cancer Metastasis Rev. 2006; 25:531540 .

12. Murugaesu N, Iravani M, van Weverwijk A, Ivetic A, Johnson DA, Antonopoulos A, Fearns A, Jamal-Hanjani M, Sims D, Fenwick K, Mitsopoulos C, Gao Q, Orr N, et al. An in vivo functional screen identifies ST6GalNAc2 sialyltransferase as a breast cancer metastasis suppressor. Cancer Discov. 2014; 4:304-317.

13. Reticker-Flynn NE, Bhatia SN. Aberrant glycosylation promotes lung cancer metastasis through adhesion to galectins in the metastatic niche. Cancer Discov. 2015; 5:168-181.

14. Storr SJ, Royle L, Chapman CJ, Hamid UM, Robertson JF, Murray A, Dwek RA, Rudd PM. The O-linked glycosylation of secretory/shed MUC1 from an advanced breast cancer patient's serum. Glycobiology. 2008; 18:456-462.

15. Singh R, Campbell BJ, Yu LG, Fernig DG, Milton JD, Goodlad RA, FitzGerald AJ, Rhodes JM. Cell surfaceexpressed Thomsen-Friedenreich antigen in colon cancer is predominantly carried on high molecular weight splice variants of CD44. Glycobiology. 2001; 11:587-592.

16. Lin WM, Karsten U, Goletz S, Cheng RC, Cao Y. Expression of CD176 (Thomsen-Friedenreich antigen) on lung, breast and liver cancer-initiating cells. Int J Exp Pathol. 2011; 92:97-105.

17. Cao Y, Merling A, Karsten U, Goletz S, Punzel M, Kraft R, Butschak G, Schwartz-Albiez R. Expression of CD175 (Tn), CD175s (sialosyl-Tn) and CD176 (Thomsen-Friedenreich antigen) on malignant human hematopoietic cells. Int J Cancer. 2008; 123:89-99.

18. Tu SM, Lin SH. Prostate cancer stem cells. Clin Genitourin Cancer. 2012; 10:69-76.

19. Engelmann K, Shen H, Finn OJ. MCF7 side population cells with characteristics of cancer stem/progenitor cells express the tumor antigen MUC1. Cancer Res. 2008; 68:2419-2426.

20. Curry JM, Thompson KJ, Rao S, Ahrens WA, McKillop IH, Sindram D. Pancreatic cancer stem cells (CSCs) express MUC1 and MUC1-expressing pancreatic cancers encompass higher levels of CSCs. In: Proceedings of the 102nd Annual Meeting of the American Association for Cancer Research; 2011 Apr 2-6; Orlando, FL. Philadelphia (PA): AACR; Cancer Res 2011;71(8 Suppl):Abstract nr 2454.

21. Stroopinsky D, Rosenblatt J, Ito K, Mills H, Yin L, Rajabi H, Vasir B, Kufe T, Luptakova K, Arnason J, Nardella C, Levine JD, Joyce RM, et al. MUC1 Is a Potential Target for the Treatment of Acute Myeloid Leukemia Stem Cells. Cancer Res. 2013; 73:5569-5579.

22. Mulholland DJ, Xin L, Morim A, Lawson D, Witte O, Wu H. Lin-Sca-1+CD49fhigh stem/progenitors are tumor-initiating cells in the Pten-null prostate cancer model. Cancer Res. 2009; 69:8555-62.

23. Yamamoto H, Masters JR, Dasgupta P, Chandra A, Popert R, Freeman A, Ahmed A. CD49f is an efficient marker of monolayer- and spheroid colony-forming cells of the benign and malignant human prostate. PLoS One. 2012; 7:e46979. 
24. Notta F, Doulatov S, Laurenti E, Poeppl A, Jurisica I, Dick JE. Isolation of single human hematopoietic stem cells capable of long-term multilineage engraftment. Science. 2011; 333:218-21.

25. Taddei ML, Parri M, Angelucci A, Bianchini F, Marconi C, Giannoni E, Raugei G, Bologna M, Calorini L, Chiarugi P. EphA2 induces metastatic growth regulating amoeboid motility and clonogenic potential in prostate carcinoma cells. Mol Cancer Res. 2011; 9:149-60.

26. Taddei ML, Parri M, Angelucci A, Onnis B, Bianchini F, Giannoni E, Raugei G, Bologna M, Calorini L, Chiarugi P. Kinase-dependent and -independent roles of EphA2 in the regulation of prostate cancer invasion and metastasis. Am J Pathol. 2009; 174:1492-1503.

27. Collins AT, Berry PA, Hyde C, Stower MJ, Maitland NJ. Prospective identification of tumorigenic prostate cancer stem cells. Cancer Research. 2005; 65:10946-10951.

28. Almogren A, Abdullah J, Ghapure K, Ferguson K, Glinsky VV, Rittenhouse-Olson K. Anti-Thomsen-Friedenreich-Ag (anti-TF-Ag) potential for cancer therapy. Front Biosci (Schol Ed). 2012; 4:840-863.

29. Glinsky VV, Huflejt ME, Glinsky GV, Deutscher SL, Quinn TP. Effects of Thomsen-Friedenreich antigen-specific peptide P-30 on beta-galactoside-mediated homotypic aggregation and adhesion to the endothelium of MDA-MB-435 human breast carcinoma cells. Cancer Res. 2000; 60:2584-2588.

30. Niehage C, Steenblock C, Pursche T, Bornhäuser M, Corbeil D, Hoflack B. The cell surface proteome of human mesenchymal stromal cells. PLoS One. 2011; 6:e20399.

31. Klammer M, Messina DN, Schmitt T, Sonnhammer EL. MetaTM - a consensus method for transmembrane protein topology prediction. BMC Bioinformatics. 2009; 10:314.

32. von Heijne G. Membrane protein structure prediction. Hydrophobicity analysis and the positive-inside rule. J Mol Biol. 1992; 225:487-494.

33. Snider C, Jayasinghe S, Hristova K, White SH. MPEx: a tool for exploring membrane proteins. Protein Sci. 2009; 18:2624-2628.

34. Krogh A, Larsson B, von Heijne G, Sonnhammer EL. Predicting transmembrane protein topology with a hidden Markov model: application to complete genomes. J Mol Biol. 2001; 305:567-580.

35. Kelly KA, Bardeesy N, Anbazhagan R, Gurumurthy S, Berger J, Alencar H, Depinho RA, Mahmood U, Weissleder R. Targeted nanoparticles for imaging incipient pancreatic ductal adenocarcinoma. PLoS Med. 2008; 5:e85.

36. Shimada K, Anai S, Fujii T, Tanaka N, Fujimoto K, Konishi N. Syndecan-1 (CD138) contributes to prostate cancer progression by stabilizing tumour-initiating cells. J Pathol. 2013; 231:495-504.

37. Mildmay-White A, Khan W. Cell surface Markers on Adipose-Derived Stem Cells: A Systematic Review. Curr Stem Cell Res Ther. 2016; 12:484-492.

38. Lee TC, Lee TH, Huang YH, Chang NK, Lin YJ, Chien PW, Yang WH, Lin MH. Comparison of surface markers between human and rabbit mesenchymal stem cells. PLoS One. 2014; 9:e111390

39. Schonberg DL, Miller TE, Wu Q, Flavahan WA, Das NK, Hale JS, Hubert CG, Mack SC, Jarrar AM, Karl RT, Rosager AM, Nixon AM, Tesar PJ, et al. Preferential Iron Trafficking Characterizes Glioblastoma Stem-like Cells. Cancer Cell. 2015; 28:441-455.

40. Shields AR, Spence AC, Yamashita YM, Davies EL, Fuller MT. The actin-binding protein profilin is required for germline stem cell maintenance and germ cell enclosure by somatic cyst cells. Development. 2014; 141:73-82.

41. Kim MJ, Lee YS, Han GY, Lee HN, Ahn C, Kim CW. Profilin 2 promotes migration, invasion, and stemness of HT29 human colorectal cancer stem cells. Biosci Biotechnol Biochem. 2015; 79:1438-1446.

42. Rittenhouse-Diakun K, Xia Z, Pickhardt D, Morey S, Baek MG, Roy R. Development and Characterization of Monoclonal Antibody to T-Antigen:(Gal $\beta 1-3$ GalNAc- $\alpha-O)$. Hybridoma. 1998; 17:165-173.

43. Yu C, Shiozawa Y, Taichman RS, McCauley LK, Pienta KJ, Keller ET. Prostate Cancer and Parasitism of the Bone Hematopoietic Stem Cell Niche. Crit Rev Eukaryot Gene Expr. 2012; 22:131-148.

44. Wang G, Wang Z, Sarkar FH, Wei W. Targeting prostate cancer stem cells for cancer therapy. Discov Med. 2012; 13:135-142.

45. Lukacs RU, Goldstein AS, Lawson DA, Cheng D, Witte ON. Isolation, cultivation and characterization of adult murine prostate stem cells. Nat Protoc. 2010; 5:702-713.

46. Hurt EM, Kawasaki BT, Klarmann GJ, Thomas SB, Farrar WL. CD44+ CD24(-) prostate cells are early cancer progenitor/stem cells that provide a model for patients with poor prognosis. Br J Cancer. 2008; 98:756-765.

47. Patrawala L, Calhoun-Davis T, Schneider-Broussard R, Tang DG. Hierarchical organization of prostate cancer cells in xenograft tumors: the CD44+alpha2beta1+ cell population is enriched in tumor-initiating cells. Cancer Res. 2007; 67:6796-6805.

48. Jiang M, Strand DW, Fernandez S, He Y, Yi Y, Birbach A, Qiu Q, Schmid J, Tang DG, Hayward SW. Functional remodeling of benign human prostatic tissues in vivo by spontaneously immortalized progenitor and intermediate cells. Stem Cells. 2010; 28:344-356.

49. Goldstein AS, Huang J, Guo C, Garraway IP, Witte ON. Identification of a cell of origin for human prostate cancer. Science. 2010; 329:568-571.

50. Lawson DA, Zong Y, Memarzadeh S, Xin L, Huang J, Witte ON. Basal epithelial stem cells are efficient targets for prostate cancer initiation. Proc Natl Acad Sci U S A. 2010; 107:2610-2615.

51. Liao CP, Adisetiyo H, Liang M, Roy-Burman P. Cancerassociated fibroblasts enhance the gland-forming capability of prostate cancer stem cells. Cancer Res. 2010; 70:7294-7303. 
52. Patrawala L, Calhoun T, Schneider-Broussard R, Zhou J, Claypool K, Tang DG. Side population is enriched in tumorigenic, stem-like cancer cells, whereas ABCG2+ and ABCG2- cancer cells are similarly tumorigenic. Cancer Res. 2005; 65:6207-6219.

53. Salvatori L, Caporuscio F, Verdina A, Starace G, Crispi S, Nicotra MR, Russo A, Calogero RA, Morgante E, Natali PG,
Russo MA, Petrangeli E. Cell-to-cell signaling influences the fate of prostate cancer stem cells and their potential to generate more aggressive tumors. PLoS One. 2012; $7: \mathrm{e} 31467$. 